A N N A L E S Annales de Bretagne et des Pays de l'Ouest

Anjou. Maine. Poitou-Charente. Touraine

108-4 | 2001

La Chambre des comptes de Bretagne

\title{
Les officiers de la Chambre des comptes de Bretagne et le corps de ville de Nantes sous l'Ancien Régime
}

Guy Saupin

\section{OpenEdition}

Journals

Édition électronique

URL : http://journals.openedition.org/abpo/1689

DOI : $10.4000 / a b p o .1689$

ISBN : 978-2-7535-1484-3

ISSN : $2108-6443$

Éditeur

Presses universitaires de Rennes

Édition imprimée

Date de publication : 20 décembre 2001

Pagination : 227-248

ISBN : 978-2-86847-674-6

ISSN : 0399-0826

\section{Référence électronique}

Guy Saupin, «Les officiers de la Chambre des comptes de Bretagne et le corps de ville de Nantes sous I'Ancien Régime ", Annales de Bretagne et des Pays de l'Ouest [En ligne], 108-4 | 2001, mis en ligne le 20 décembre 2003, consulté le 02 mai 2019. URL : http://journals.openedition.org/abpo/1689 ; DOI : 10.4000/abpo. 1689 


\title{
Les officiers de la Chambre des comptes de Bretagne et le corps de ville de Nantes sous l'Ancien Régime
}

\author{
Guy SAUPIN \\ professeur d'histoire moderne \\ CRHMA-Université de Nantes
}

Même si, par son édit de 1547, le roi Henri II a prétendu établir une incompatibilité entre les offices de justice royale et les responsabilités électives municipales, la réaction quasi générale des villes du royaume, qui ont obtenu les unes après les autres de se faire exempter de cette obligation, a au contraire facilité la montée en puissance du milieu robin dans la vie municipale, souvent au détriment de l'influence de l'élite marchande ${ }^{1}$. Dans la vie municipale nantaise, alors que les parlementaires ont réglé leur conduite dans le sens de l'édit royal incriminé, les officiers des comptes n'ont pas craint de s'associer aux autres strates de l'oligarchie urbaine ${ }^{2}$ pour s'intégrer au bureau de ville composé d'un maire et de dix échevins à la création de 1565, mais ramenés à six pour des questions de prestige et de facilité de recrutement à partir de 1584. De 1569 à 1675 pour le poste de maire et de 1573 à 1664 pour les postes d'échevins, les officiers de la chambre des comptes ont ainsi tenu leur place dans la gestion de la communauté urbaine, avant qu'un divorce complet et définitif ne soit prononcé.

De 1565 à 1598, les maire et échevins de Nantes sont élus directement par une assemblée générale non formalisée juridiquement, mais obéissant dans la pratique sociale à l'idéal représentatif de l'expression de la sanior pars. À la reddition du duc de Mercour, cette entière liberté est perdue, Henri IV ayant désiré sanctionner symboliquement la dernière ville ligueuse de son royaume, même si l'essentiel des privilèges a été maintenu par un roi de paix à la recherche de la réconciliation de l'oligarchie urbaine. Dorénavant, jusqu'en 1789, la ville de Nantes voit s'appliquer la règle de la désignation de trois candidats pour un poste, comme bien d'autres villes

1. Bernard Chevalier, Les bonnes villes de France du XIV au XVI siècle, Paris, Aubier, 1982 , p. $56-63$ et $143-149$.

2. Guy SaUPIN, « Sociologie du corps de ville de Nantes sous l'Ancien Régime, 15651789 ", Revue Historique, 1996, CCXCV-2, p. 299-331. 
du royaume ${ }^{3}$. Des listes d'éligibilité sont composées selon le principe de cooptation par les édiles anciens et en fonction, et l'assemblée générale des électeurs, toujours sans règlement, classe les candidats parmi lesquels le gouverneur sélectionne les noms qui doivent logiquement, à moins que d'autres hautes protections ne s'avèrent plus décisives, être retenus par le secrétaire d'État chargé de la Bretagne ${ }^{4}$.

À partir de ce système, il apparaît fort instructif d'apprécier la place accordée aux officiers de la cour souveraine provinciale installée à Nantes dans la proposition légale envoyée au pouvoir royal, mais surtout dans une comparaison entre l'idéal nantais, exprimé dans le premier suffragé pour le poste de maire ou les deux premiers pour ceux d'échevins, et le choix définitif de la monarchie. Dans les trois étapes décisives - cooptation, classement et nomination royale - peuvent s'exprimer des recommandations plus ou moins appuyées d'autorités comme l'évêque, le gouverneur, le premier président de la chambre des comptes, le sénéchal, le président du présidial et l'action de coalitions des familles très liées à l'administration de la ville, sorte de noyau dur de l'oligarchie municipale ${ }^{5}$. Tout tourne normalement autour du représentant direct de la monarchie, le gouverneur puis l'intendant après 1689, le premier ne cessant pourtant jamais d'être considéré comme le tuteur politique officiel du corps de ville. Dans l'arbitrage rendu, le poids de l'auto-régulation locale est loin d'être négligeable.

Dans le cadre de l'étude sociale des institutions, cette contribution entend d'abord replacer les officiers des Comptes ${ }^{6}$ dans les grandes phases de l'évolution sociologique du corps de ville nantais, avant de s'intéresser à l'environnement familial de ces officiers royaux supérieurs concernés par la vie municipale afin de tenter de dégager quelques remarques sur leurs profils lignagers et sur les caractéristiques de l'ascension sociale urbaine aux XVI ${ }^{\mathrm{e}}$ et XVII ${ }^{\mathrm{e}}$ siècles.

\section{Les officiers des comptes élus maires de Nantes}

De 1565 à 1598, phase d'implantation laborieuse du nouveau type de municipalité, période pendant laquelle le lobby marchand et les coalitions de familles ayant joué un rôle essentiel dans le précédent conseil des bour-

3. Annette Finley-Croswhite, "Henri IV et les villes » dans Colloque Pau-Nérac, Henri IV, le roi et la reconstruction du royaume, Pau, 1990, p. 195-205; Idem, Henry IV and the Towns, Cambridge, cuP, 1999.

4. Guy SAUPIN, Nantes au XVII siècle. Vie politique et société urbaine, 1598-1720, Rennes, PUR, 1996, p. 75-108. Utiliser l'index des noms de familles.

5. Guy SAUPIN, « Les oligarchies municipales en France sous l'Ancien Régime : réflexion méthodologique sur l'analyse historique de leur reproduction à partir de l'exemple de Nantes ", dans Claude PETITFRERE (éd.), Construction, reproduction et représentation des patriciats urbains de l'Antiquité au XXe siècle, Tours, CEHVI, 1999, p. 95-112.

6. Pour situer tous les officiers cités dans cette étude, consulter le meilleur instrument actuellement disponible : Gaëtan d'AVIAU DE TERnAY, Dictionnaire des Magistrats de la Chambre des comptes de Bretagne, dact., Paris, 1995. 
geois forment certainement le groupe de pression le plus influent ${ }^{7}, 23$ mai- $^{\text {- }}$ res ont présidé le bureau de ville. L'élite marchande en fournit neuf, la chambre des comptes quatre auxquels s'ajoute le général des finances Myron, le présidial quatre, le parlement de Bretagne deux. L'ensemble est complété par deux avocats et un grand maître des eaux et forêts dont l'essentiel de la carrière a été marchande.

Tableau $n^{\circ} 1$ - Les officiers des comptes maires de Nantes (1565-1598)

\begin{tabular}{|l|c|c|c|c|}
\hline Dates & Maires & Offices & $\begin{array}{c}\text { Situation } \\
\text { antérieure }\end{array}$ & $\begin{array}{c}\text { situation } \\
\text { postérieure }\end{array}$ \\
\hline $1569-1570$ & Pierre Cornulier & Maître & $\begin{array}{c}\text { Sec. gouv. Bretagne } \\
\text { Md., Rec. fouages }\end{array}$ & Trés. de France \\
1573 & Michel Le Lou & Maître & Avocat, PS ville & Prévôt \\
$1590-1591$ & Pierre André & Av. général & $\begin{array}{c}\text { Avón } \\
\text { Militaire, Auditeur }\end{array}$ & \\
\hline
\end{tabular}

Ainsi, aucun président aux Comptes n'est devenu maire dans ce dernier tiers du Xvi ${ }^{\mathrm{e}}$ siècle. Sans doute mesurons-nous ici les retombées de l'opposition des cours de justice à la transformation du conseil des bourgeois en bureau de ville et de l'hostilité déclarée du présidial et de la prévôté envers la nouvelle institution jusqu'en 1575 et 1581. La cour souveraine nantaise se montre plutôt solidaire, par la conduite de ses officiers supérieurs, des autres compagnies officières, bien que peu affectée par la redistribution des compétences en matière de police urbaine. Néanmoins, par l'intermédiaire des promotions politiques de maîtres, elle normalise beaucoup plus vite ses relations avec la nouvelle municipalité. Pour ce faire, les parcours individuels comptent beaucoup.

Pierre Cornulier, quatrième maire de Nantes après deux marchands et un avocat ${ }^{8}$, est poussé par le gouverneur de Bretagne dont il est le secrétaire, ce représentant du roi étant chargé de la bonne application de la politique royale, alors que le capitaine du château, le comte de Sanzay, se montre particulièrement récalcitrant jusqu'à sa démission en 1580. L'élection de Michel Le Lou ${ }^{9}$ en 1573 est autant un succès pour le lobby marchand, dont il est l'un des plus illustres représentants ${ }^{10}$, qu'un signe du ralliement des Comptes, puisqu'il est d'abord élu échevin et sous-maire en tant que commerçant d'envergure, en 1571, avant d'acquérir l'office de maître dans lequel il est reçu le 22 octobre 1572.

7. Guy SAUPIN, « Les marchands dans le Conseil des bourgeois de Nantes au milieu du xvl siècle ", dans Mélanges Jean Tanguy, Bretagnes, art, négoce et société de l'Antiquité à nos jours, Brest, 1996, p. 229-239.

8. Stéphane de La Nicolliere-Teijeiro, Alexandre Perthuis, Le Livre Doré de l'Hôtel de ville de Nantes, Nantes, 1873, t. 1, p. 137-138; Gaëtan d'AviAu DE TERnAY, op. cit., p. 101.

9 . Arch. dép. de Loire-Atlantique, $25 \mathrm{~J}$ Nantes 11. Arch. mun. de Nantes, Notice généalogique sur la famille Le Lou et ses alliances, Extrait de Revue de Bretagne, 1911, p. 186; Gaëtan d'AVIAU DE TERnAY, op. cit., p. 247-248; LAPEYRE, Henri, Une famille de marchands, les Ruiz, Paris, 1955, p. 60-64, 89, 92, 147.

10. Arch. mun. de Nantes, BB 4, f. 19, 62, 66, 195, 205, 216, 242, 272, 292, 392, 402, 410. 
Les deux dernières élections sont très liées au basculement de Nantes dans la rébellion ligueuse derrière le duc de Mercœur ${ }^{11}$, mais la possession de l'office des comptes ne revêt pas la même signification. Pierre André12, déjà avocat général avant l'éclatement de la crise en 1589, est appelé au poste de maire pour ses convictions ligueuses qui en font un membre de la garde rapprochée du couple Mercœur. À sa sortie de mairie, il délaisse la chambre des comptes pour prendre la charge de prévôt ${ }^{13}$, très complémentaire du bureau de ville en ce qui concerne la tenue conjointe du tribunal de police depuis le compromis passé en 1581. La famille André ${ }^{14}$ est par ailleurs très liée à la vie municipale dans le second $\mathrm{xvI}^{\mathrm{e}}$ siècle puisque son père, Mathieu, avocat, avait été très actif dans le conseil des bourgeois avant d'être désigné troisième maire de Nantes en 1568, et que lui-même est passé par les charges d'échevin et de procureur du roi syndic (novembre 1583 - septembre 1586) avant de devenir maire.

Jean Fourché ${ }^{15}$, auditeur à la chambre depuis 1583, s'est vu récompensé de sa fidélité à Mercœur par l'obtention d'une charge de maître dans la nouvelle cour des comptes créée par le duc pour contrer le transfert de l'ancienne vers Rennes sur ordre royal. Échevin de juillet 1587 à avril 1590, il a pris en mains, comme sous-maire, le bureau de ville lorsque la duchesse de Mercœur a fait emprisonner le maire Charles de Harouys ${ }^{16}$ en avril 1589. Très précieux dans l'organisation militaire tant dans le domaine de l'artillerie en Bretagne que dans la rénovation des fortifications nantaises, il est rappelé comme maire en 1597 à un moment où la plupart des autres villes ligueuses se sont déjà ralliées à Henri IV et où le duc et l'oligarchie ligueuse jouent leur va-tout en misant sur une défaite des troupes royales devant les tercios espagnols sur le front de Picardie, spécialement lors de la surprise d'Amiens ${ }^{17}$. Pour Fourché, l'élévation dans la chambre a bien représenté une récompense pour son engagement idéologique, mais l'office a pu être conservé lors de la réinstallation de la cour loyaliste à Nantes en 1598.

En 1598, le roi Henri IV imposant le contrôle royal sur le renouvellement du corps de ville, la comparaison des préférences affichées peut commencer. En ce qui concerne la direction du bureau de ville au XvII ${ }^{\mathrm{e}}$ siècle

11. Dominique LE PAGE, "Le personnel de la Chambre des comptes de Bretagne en conflit (années 1589-1591)", Cahiers d'histoire, tome 4, n 4, 2000, p. 587-609.

12. Stéphane de La Nicolliere-TeiJeIRo, Alexandre Perthuis, op. cit., p. 134, 141, 169, 180181 ; Gaëtan d'Aviau de TERnaY, op. cit., p. 3.

13. Charles-Antoine CARDOT, Le Parlement de la Ligue en Bretagne, Thèse de droit, Rennes, 1964, t. 3, p. 730-742.

14. Arch. dép. de Loire-Atlantique, 25 J Nantes 1. Arch. mun. de Nantes, CC 338, DD 15, FF 30. Mathieu André et son épouse servent d'intermédiaire pour l'achat de l'hôtel de Bizart destiné à devenir l'Hôtel de ville (1574-1578) ; Jacques VAILHEN, Le conseil des bourgeois de Nantes, Thèse de droit, Rennes, 1965, tome 3.

15. René KERVILER, Répertoire général de bio-bibliographie bretonne, Rennes, 1908, t. 14, p. 306-307; Gaëtan d'AVIAU DE TERnAY, op. cit., p. 142; P. LEVOT (dir.), Biographies bretonnes, Paris, 1852, t. 1, p. 725.

16. Guy SAUPIN, Nantes au XVII siècle, op. cit., p. 455.

17. Idem, Nantes au temps de l'édit, La Crèche, Geste Éditions, 1998, p. 186-208. 
(1598-1719), les officiers des Comptes s'inscrivent en seconde position, loin derrière l'imposante suprématie exercée par l'ensemble de la sénéchaussée et du présidial, mais toujours devant une représentation marchande dont l'importance varie beaucoup selon les points de vue.

Tableau $n^{\circ} 2$ - Importance relative des officiers des Comptes dans la sélection au poste de maire de Nantes au XVII siècle (1598-1719)

\begin{tabular}{|l|c|c|c|}
\hline Expressions (\%) & Comptes & Présidial & Marchandise \\
\hline Trois meilleurs scores & 19 & 59,5 & 16,5 \\
Le premier suffragé & 21,9 & 69,8 & 4,1 \\
Le choix royal & 22 & 60 & 8 \\
\hline
\end{tabular}

Il convient de souligner la remarquable convergence qui se dégage sur la place à accorder aux officiers de la cour souveraine, tandis que le pouvoir central corrige aussi bien un élan jugé excessif envers le présidial qu'un manque de confiance dans l'élite marchande dont les représentants ne sont souvent proposés qu'en seconde ou troisième position.

\section{Tableau $\mathbf{n}^{\circ} 3$ - Types d'officiers soutenus par les Nantais et la monarchie (1598-1719)}

\begin{tabular}{|l|c|c|}
\hline Préférence du corps politique & Choix royal \\
\hline Présidents & 10 & 5 \\
Maîtres & 2 & 4 \\
Auditeurs & 2 & 1 \\
Avocats généraux & 2 & 1 \\
Total & $16(21,9 \%)$ & $11(22 \%)$ \\
\hline
\end{tabular}

Il existe bien un respect global de la hiérarchie des charges, donc de la puissance sociale des lignages qui les tiennent, même si le pouvoir s'accorde une certaine liberté en assurant une réelle promotion des maîtres. Ces élévations sont quasiment toutes placées dans les deux premiers tiers du XVII ${ }^{\mathrm{e}}$ siècle, illustrant bien en cela l'opposition entre les deux grandes phases historiques déjà évoquées, le règne de Louis XIV jusqu'à la création des offices municipaux apparaissant comme une période de transition où les initiatives locales et centrales ne sont pas toujours bien coordonnées.

Dans la concertation établie entre le corps politique nantais et le pouvoir royal, les officiers des Comptes bénéficient d'une bonne appréciation mutuelle qui leur assure un taux de réussite très élevé. Seul trois notables placés en première position ne sont pas parvenus à faire confirmer ce succès auprès du gouvernement royal. Guillaume Raoul paie peutêtre un net engagement ligueur derrière le duc de Mercour ${ }^{18}$, Henri IV inscrivant plutôt sa politique de réconciliation de l'oligarchie nantaise dans

18. Gaëtan d'Aviau de Ternay, op. cit., p. 345; Barthélémy Pocquet, Histoire de Bretagne, Rennes, 1913, t. 5, p. 187-190, 223, 231, 236, 274, 279, 283. 
l'échevinage, en réservant nettement la direction du corps de ville aux familles demeurées loyalistes pendant la guerre civile ${ }^{19}$. Cette prudence a dû être d'autant plus marquée que l'élévation du premier maire ancien ligueur (1607-1609), en la personne de Michel Loriot, sénéchal des régaires, a correspondu en 1608 à l'éclatement d'un conflit autour de l'acquittement de la taxe du liard pour livre, une des sources des octrois urbains, sans que le maire y soit d'ailleurs pour quelque chose étant donné ses origines socioprofessionnelles.

\section{Tableau $n^{\circ} 4$ - Tableau des officiers proposés ou retenus comme maires de Nantes}

\begin{tabular}{|l|c|c|c|}
\hline Dates & Noms & Offices & Sélection \\
\hline $1603-1605$ & Yves Le Lou & Maître & Nommé \\
1609 & Guillaume Raoul & Président & Proposé \\
$1615-1617$ & Pierre Bernard & Président & Nommé \\
$1623-1625$ & Louis de Harouys & Président & Nommé \\
$1629-1631$ & René de La Tullaye & Maître & Nommé \\
$1642-1644$ & Christophe Juchault & Président & Nommé \\
$1644-1647$ & Yves de Monti & Maître & Nommé \\
$1648-1650$ & Mathurin Boux & Maître & Nommé \\
$1657-1659$ & René de Pontual & Président & Nommé \\
$1659-1661$ & Jacques Huteau & Président & Nommé \\
1671 & Sébastien de Pontual & Président & Proposé \\
$1673-1675$ & Jean Regnier & Auditeur & Nommé \\
1688 & Jean-Baptiste de Cornulier & Président & Proposé \\
$1690-1692$ & Pierre Noblet & Av. général & Nommé \\
\hline
\end{tabular}

L'échec de Sébastien de Pontual ${ }^{20}$ est à replacer dans le cadre de la relance d'une ambitieuse politique mercantiliste imprimée par Colbert depuis 1664. Soucieux d'attirer les capitaux du second ordre dans les sociétés commerciales monopolistiques, le ministre soutient le discours étatique de l'honorabilité du commerce en appuyant davantage les candidats marchands suggérés par les Nantais. Gratien Libault, le rival préféré, est ainsi un des principaux marchands au long cours ${ }^{21}$, le chef d'une famille très orientée dans la grande pêche à la morue et l'un des principaux artisans de l'ouverture de la route antillaise dans les années 1640.

19. Guy SAUPIN, «Le corps de ville de Nantes sous Henri IV (1598-1610), outil de réconciliation politique ", dans Jean-Pierre BARDET et al., État et société en France aux XVII et XVII siècles, Paris, PUPS, 2000, p. 489-504.

20. Henri Frotier de La Messeliere, Filiations bretonnes, Saint-Brieuc, 1924, t. 4, p. 434; Gaëtan d'Aviau DE Ternay, op. cit., p. 333.

21. Guy SAUPIN, "Les marchands nantais et l'ouverture de la route antillaise, 1639-1650 ", dans Jean-Pierre SANCHEz (éd.), Dans le sillage de Colomb. L'Europe du ponant et la découverte du Nouveau Monde (1450-1650), Rennes, PUR, 1995, p. 178-179; idem, Nantes au XVII siècle, op. cit., p. 456; Christelle LAUCOIN, La naissance du trafic antillais (1638-1660), mémoire de maîtrise, université de Nantes, 1999, p. 83-86. 
En 1688, l'oubli du président Jean-Baptiste de Cornulier au profit de Paul Cassard $^{22}$, simple juge criminel au présidial, est plus difficile à interpréter en terme de stratégie politique. L'affaire est d'autant plus surprenante qu'elle affecte un membre d'un lignage prestigieux ayant donné deux maires à la ville en 1569-1570 et 1605-1607, avant de s'imposer comme l'une des dynasties les plus influentes dans le parlement de Bretagne ${ }^{23}$. Même si l'impétrant appartient à une branche latérale, le prestige global de cette famille ne peut jouer qu'en sa faveur. La carrière de ce serviteur de l'État monarchique n'est affectée d'aucun incident qui aurait pu entraîner une disgrâce. Successivement militaire, conseiller au parlement en 1664, président aux comptes (1674-1698), son travail comme commissaire royal à la réformation du domaine en 1678 a donné satisfaction puisqu'il est promu au conseil d'État en 1685.

Un tel parcours aurait dû, au contraire, avantager le président de Cornulier puisque s'offrait l'occasion de renouer symboliquement les liens sociaux quasiment évanouis entre les officiers des comptes et le corps de ville depuis l'amputation des privilèges d'anoblissement municipal et la mise sous contrôle de la gestion financière de la ville par les commissaires royaux décidées par Colbert. Il n'est pas possible d'invoquer la mauvaise réputation jansénisante de la famille puisqu'il ne s'agit pas d'un membre de la branche suspecte et que la grande crise n'est pas encore réouverte. Restent à supputer soit une stratégie royale visant à ne pas confier la présidence du bureau de ville à des représentants de grandes familles historiques jugés trop indépendants ${ }^{24}$, soit un désengagement du président luimême, placé là contre son gré par le corps politique.

\section{Les officiers des comptes élus à l'échevinage nantais}

Dans le dernier tiers du XvI ${ }^{\mathrm{e}}$ siècle, le recrutement des échevins n'obéit pas à la même logique que celui des maires. Alors que l'élite marchande ne fournit qu'environ $40 \%$ de ces derniers, elle représente $62 \%$ des premiers. Les milieux professionnels qui entrent en compétition avec le commerce maritime et la vente des produits de luxe sont plutôt les professions juridiques secondaires (17\%) comme les avocats (8\%), les procureurs (4\%), les notaires (3\%) et les juges des régaires ( $2 \%)$, mais aussi les officiers de

22. Arch. dép. de Loire-Atlantique, 25 J Nantes 4; Gaëtan d'AVIAU DE TERNAY, op. cit., p. 7980. Il convient d'adopter une attitude fort critique sur les notices rédigées il y a un siècle sur cette famille qui a obtenu une reconnaissance d'ancienne noblesse dans un arrêt du conseil de 1702; René KERVILER, op. cit., t. 8, p. 80-86. Et surtout Régis de L'EsTOURBEILLON, La noblesse de Bretagne, Vannes, 1895, t. 2, p. 211-212.

23. Gaëtan d'Aviau de Ternay, op. cit., p. 102; René Kerviler, op. cit., t. 10, p. 290-303; Frédéric SAULNIER, Le parlement de Bretagne, 1554-1790, Rennes, 1909, t. 1, p. 262-267.

24. Cela pourrait ainsi préfigurer l'élection de 1716 où le pouvoir royal écarte le sénéchal Louis Charette, issu d'une dynastie ayant déjà donné sept maires au XVII ${ }^{\mathrm{e}}$ siècle, au profit du procureur du roi au présidial André Boussineau, déjà utilisé comme subdélégué temporaire dans la première décennie du XVIII ${ }^{\mathrm{e}}$ siècle; Guy SAUPIN, Nantes au XVII siècle, op. cit., p. 451 . 
finances (11\%) dont beaucoup de receveurs de fouages. Les officiers des cours de justice royale les plus en vue ne forment qu'une infime minorité composée essentiellement d'officiers des comptes (7\%) contre un seul du présidial. Cette dernière répartition indique clairement que ce n'est pas le manque de prestige de la fonction municipale qui influe, mais bien plutôt la radicalité de l'opposition des divers corps judiciaires à la réforme municipale de 1559-1565.

Tableau $n^{\circ} 5$ - Les officiers des comptes élus comme échevins au XVIe siècle

\begin{tabular}{|l|c|c|c|c|}
\hline Mandats & Échevins & Sieuries & Offices & Autres charges \\
\hline $1573-1575$ & Bernard de Monti & & Maître & \\
$1573-1575$ & Aymé Adam & Tartifume & Auditeur & \\
$1574-1576$ & François Simon & La Folliette & Auditeur & Greffier Présidial \\
$1583-1585$ & Jean Cousin & La Roche & Auditeur & Rec. gén. Ligue \\
$1584-1586$ & Étienne Maillard & La Minguaye & Auditeur & \\
$1587-1590$ & Jean Fourché & La Courosserie & Auditeur & Maître, Maire \\
$1589-1591$ & Jean de La Tullaye & La Jaroussaye & Auditeur & Maître \\
\hline
\end{tabular}

L'intégration d'officiers des Comptes dans l'échevinage s'est faite en deux vagues. La première débute huit ans après la création effective du bureau de ville, les premières années étant marquées par l'action déterminée du lobby marchand, soutenu par quelques officiers de finances. Cette précocité du ralliement s'explique peut-être par l'influence d'une filière menant du commerce à la chambre via les finances. Ainsi Guillaume Gougeon, "banquier des nobles bourgeois ", désigné comme échevin en 1568 , acquiert-il un office d'auditeur pendant son mandat en $1569^{25}$. De même, Michel Le Lou, descendant d'une importante famille marchande, lui même actif dans le commerce tout en tenant l'office de receveur des fouages de l'évêché de Tréguier, élu échevin et sous-maire en 1571, négocie un office de maître en octobre 1572, juste avant d'être élu maire de la ville en 1573. Une seconde vague semble relancée par la normalisation générale des relations entre toutes les institutions nantaises en 1580-1581.

Il est significatif d'observer que le premier pas est effectué par un officier dont la famille n'a pas d'enracinement lointain à Nantes. Bernard de Monti est un noble florentin, immigré depuis 25 à 30 ans dans la ville ${ }^{26}$, après avoir sans doute suivi les Strozzi dans la suite de Catherine de Médicis, épouse du roi Henri II. À l'exception de cet exemple, tous les promus sont titulaires d'offices d'auditeurs, charges inférieures de la cour souveraine. La coupure fonctionnelle et honorifique qui traverse la compagnie des comptes a donc bien sa traduction sociale dans ces lignages en ascension, clivage nettement mis en lumière par la répartition des rôles au sein

25. Gaëtan d'Aviau DE TeRnAY, op. cit., p. 172.

26. Arch. dép. de Loire-Atlantique, B 53, $\mathrm{f}^{\circ}$ 81. Lettres de naturalité (décembre 1548-mai 1549) qui reconnaissent une résidence nantaise de plus de deux ans; Gaëtan d'AvIAU DE TERNAY, op. cit., p. 301. 
du corps de ville. Les quelques mutations postérieures relevées se rattachent toutes aux opportunités offertes par la grande crise ligueuse ${ }^{27}$.

Avec le XvII ${ }^{\mathrm{e}}$ siècle, cette distinction entre offices supérieurs et inférieurs est encore plus marquée puisque, en dehors des auditeurs, l'échevinage ne s'ouvre plus qu'aux officiers auxiliaires dont le prestige et la valeur de la charge sont nettement ramenés vers le bas, les incluant dans cet ensemble d'officiers de judicature moyens et inférieurs ${ }^{28}$ qui disputent alors les postes aux grands marchands et aux meilleurs détaillants de produits de luxe. L'absence de maître et surtout l'entrée de trois greffiers marquent ainsi la perte de considération dont est victime la magistrature municipale seconde au sein de l'élite robine nantaise.

Tableau $n^{\circ} 6$ - Les officiers des comptes élus échevins au XVII ${ }^{e}$ siècle

\begin{tabular}{|c|c|c|c|c|c|}
\hline Mandats & Échevins & Sieuries & Offices & $\begin{array}{c}\text { Situation } \\
\text { antérieure }\end{array}$ & $\begin{array}{c}\text { Situation } \\
\text { postérieure }\end{array}$ \\
\hline 1598-1601 & G. Marcel & Maupas & Auditeur & \multirow{10}{*}{$\begin{array}{c}\text { Banquier } \\
\text { Greffe Cptes }\end{array}$} & \multirow{14}{*}{$\mathrm{M}^{\mathrm{e}}$ Hôtel SM } \\
\hline 1601-1604 & M. Juchault & La Bourderie & Auditeur & & \\
\hline $1604-1607$ & P. Monnier & La Fresnaye & Auditeur & & \\
\hline $1605-1608$ & P. Davy & Chesne Moreau & Auditeur & & \\
\hline $1611-1614$ & A. Le Feuvre & Pont & Auditeur & & \\
\hline $1618-1621$ & F. Grandamy & & Auditeur & & \\
\hline $1623-1626$ & P. Madeleneau & La Ménardière & Auditeur & & \\
\hline $1624-1627$ & J. Le Breton & La Rouxelière & Greffier & & \\
\hline $1625-1628$ & P. Le Masle & Juigné & Auditeur & & \\
\hline $1636-1637$ & J. Ragaud & Perrières & Auditeur & & \\
\hline $1637-1639$ & M. Gallinière & & Auditeur & \multirow{6}{*}{ G.livres Ch. } & \\
\hline $1645-1648$ & C. Juchault & Perron & Auditeur & & \\
\hline $1645-1648$ & D. Barillier & Saz & Auditeur & & \\
\hline $1646-1649$ & G. Macé & Lousselière & Greffier & & \\
\hline $1651-1654$ & J. Regnier & La Souchais & Auditeur & & $\begin{array}{c}\text { Maire } \\
1673-1675\end{array}$ \\
\hline 1661-1664 & M. Forcheteau & & Greffier & & \\
\hline
\end{tabular}

La répartition par décennie se révèle fort irrégulière, mais souligne surtout le décrochage rapide qui s'opère dans la seconde moitié du XVII ${ }^{\mathrm{e}}$ siècle, dans une transition d'une quinzaine d'années qui voit disparaître un comportement hérité du dernier tiers du xvie siècle. La rupture entre l'échevinage et les officiers des Comptes, consommée très rapidement au début du règne personnel de Louis XIV, ne doit pas être comprise comme une exclusion d'une compagnie victime de la montée en puissance d'un groupe social concurrent, mais comme une désertion volontaire d'un milieu

27. Il s'agit des promotions de Jean Fourché et de Jean de La Tullaye.

28. Michel CASSAN (éd.), Les officiers moyens à l'époque moderne, France, Angleterre, Espagne, Limoges, PULIM, 1998; idem (éd.), Officiers moyens (I), Cahiers du Centre de Recherches Historiques, octobre 1999, nº 23. 
qui porte maintenant un jugement négatif sur la réputation de l'institution municipale. Même si la chronologie observable fait coïncider le début du malaise avec la période de la Fronde, le véritable divorce est prononcé lorsque Colbert supprime le privilège d'anoblissement échevinal en 1667. Pour des lignages engagés dans le processus d'intégration dans le second ordre par l'anoblissement graduel sur deux générations offert par les offices de la cour souveraine, il devenait totalement inopportun de brouiller leur image extérieure en intégrant une institution qui se voyait amputée d'un rôle social de même type.

La rupture aurait même pu se produire un peu plus tôt sans une réforme électorale temporaire imaginée par le maréchal de La Meilleraye, lieutenant général en Bretagne et gouverneur de Nantes et de son comté. En 1644, le représentant du roi fit accepter le principe de trois listes d'éligibilité pour les catégories de postes à pourvoir : une première composée de membres de la chambre des comptes renforcés des officiers de finances, une seconde des membres du présidial rejoints par les autres officiers inférieurs de justice et une troisième des marchands. Après avoir servi pendant dix ans, le système fut abandonné sur ordre du maréchal, sensible aux difficultés d'inscription des candidats ne relevant ni des offices, ni de la marchandise. Si quatre notables ont bénéficié de cet effet structurel, l'impulsion s'est surtout fait sentir en tout début de période avant de s'étioler rapidement, victime sans doute de la réaction aristocratique saisissant la compagnie.

\section{Tableau $n^{\circ} 7$ - Comparaison entre les choix nantais et les nominations royales}

\begin{tabular}{|l|c|c|c|c|}
\hline Phases & Origine & $\begin{array}{c}\text { Les six } \\
\text { proposés }\end{array}$ & $\begin{array}{c}\text { Les deux } \\
\text { premiers }\end{array}$ & $\begin{array}{c}\text { Choix } \\
\text { royal }\end{array}$ \\
\hline \multirow{2}{*}{$1598-1639$} & Comptes & $17-9,14 \%$ & $10-16,13 \%$ & $11-11,96 \%$ \\
& Présidial & $38-20,43 \%$ & $33-53,23 \%$ & $19-20,65 \%$ \\
& Av. et Proc. & $29-15,59 \%$ & $03-4,84 \%$ & $12-13,04 \%$ \\
& Marchands & $82-44,08 \%$ & $11-17,74 \%$ & $30-32,60 \%$ \\
\hline \multirow{2}{*}{$1639-1668$} & Comptes & $22-12,57 \%$ & $06-15,79 \%$ & $05-8,62 \%$ \\
& Présidial & $21-12 \%$ & $08-21,06 \%$ & $09-15,52 \%$ \\
& Av. et Proc. & $43-24,57 \%$ & $12-31,58 \%$ & $10-17,24 \%$ \\
& Marchands & $70-40 \%$ & $06-15,79 \%$ & $28-48,27 \%$ \\
\hline \multirow{2}{*}{$1669-1699$} & Comptes & 0 & 0 & 0 \\
& Présidial & $02-1,19 \%$ & 0 & 0 \\
& Av. et Proc. & $57-33,93 \%$ & $22-39,28 \%$ & $19-31,14 \%$ \\
& Marchands & $88-52,38 \%$ & $30-53,57 \%$ & $37-60,65 \%$ \\
\hline
\end{tabular}

Dans la seconde colonne, seulement 19 élections dans la période 1639-1668, à cause de la réforme de La Meilleraye.

Ce tableau de synthèse, qui compare non seulement les trois expressions politiques pour la désignation annuelle de deux échevins, mais aussi les variations de ce choix pour les quatre grands milieux socioprofession- 
nels pourvoyeurs, illustre l'importance des évolutions qui ont affecté le XVII ${ }^{\mathrm{e}}$ siècle. Dans les quatre premières décennies, la représentation des Comptes est celle qui connaît le moins de variations entre les deux formes d'expression nantaise et entre l'idéal nantais et la décision finale du pouvoir royal. La correction monarchique, car elle existe néanmoins, tend à atténuer le résultat qu'aurait donné un suffrage libre (11,96\% contre $16,13 \%)$, en se rapprochant nettement du niveau de la sélection globale $(9,14 \%)$. Cette modification à la baisse reste toutefois beaucoup moins sévère que celle qui affecte le présidial très survalorisé par le corps politique nantais et, dans une moindre mesure, que la réévaluation du poids des avocats et procureurs ou des marchands. Dans une phase historique marquée par le souvenir de la grande rébellion anti-monarchique de 15891598 , où la communauté place toute sa confiance dans les officiers des principales cours de justice royale, les auditeurs aux comptes représentent une minorité appréciée de l'échevinage.

Dans la période 1640-1668 où la monarchie contracte volontiers l'importance relative des gens de justice pour en faire profiter les marchands, les officiers des comptes n'apparaissent plus comme les plus protégés par la monarchie. Le résultat final $(8,62 \%)$ est non seulement très inférieur à l'expression de l'idéal nantais (15,79\%), mais également à cette évaluation toujours plus modérée donnée par les six proposés (12,57 \%), ce qui établit une différence d'attitude avec les officiers du présidial ou les avocats et procureurs pour lesquels est établi un positionnement intermédiaire. Le processus de raréfaction des officiers des Comptes semble donc enclenché par une certaine réticence du pouvoir royal dont l'interprétation reste malaisée puisque c'est au même moment que le maréchal de La Meilleraye établit sa réforme de l'éligibilité qui est de sens contraire. Le malaise grandissant entre une monarchie s'appuyant prioritairement sur des commissaires et des fermiers pour ses finances et nombre de compagnies d'officiers, dont les porte-parole sont les cours souveraines et spécialement les parlements, a sa part de responsabilité.

Ce moindre soutien et un effet de mimétisme avec l'attitude des parlementaires qui, depuis l'édit de Henri II de 1547, refusent de s'intégrer directement aux corps de ville, se conjuguent pour dissoudre fort rapidement les liens unissant jusque là les auditeurs et les responsabilités échevinales. Le gouverneur, puis l'intendant ne peuvent que constater qu'à partir de 1669 les édiles et les électeurs ne leur proposent plus d'officiers inférieurs des Comptes, parce que ces derniers refusent d'entrer dans le jeu de la cooptation, entraînant même derrière eux les officiers au présidial qui vivent encore accrochés au grand mythe de l'alignement de leur siège sur les cours souveraines quant au privilège d'anoblissement ${ }^{29}$.

29. Guy SAUPIN, « Fonctionnalisme urbain et sociologie des corps de villes en France aux Temps modernes " dans Guy SAUPIN (éd.), Le pouvoir urbain dans l'Europe atlantique aux Temps modernes, Table ronde Nantes, 2000, Actes à paraître. 


\section{L'environnement familial des officiers des comptes nommés au bureau de ville}

Afin de mieux cerner le profil des familles de ces officiers qui ont tenté et qui ont réussi à intégrer le bureau de ville de 1565 à 1675, il convient de reconstituer autant que faire se peut leur identité lignagère aussi bien dans une logique verticale intergénérationnelle ascendante que dans une dimension horizontale en pointant tous les parents proches sur une même génération. Les données collectées ont été synthétisées dans plusieurs tableaux qui sont ici présentés.

Dans toutes ces familles en ascension sociale, le profil varie bien sûr selon la qualité de l'office détenu lors de l'accession au poste de maire. Sur les quinze présidents du bureau de ville, on relève sept maîtres, cinq présidents, deux avocats généraux et un seul auditeur. Les nominations de l'auditeur Jean Regnier ${ }^{30}$ en 1673 , qui retrouve la direction du corps de ville après une expérience échevinale (1652-1655), et de l'avocat général Pierre Noblet $^{31}$ en 1690 témoignent plus de la perte de prestige de la fonction auprès de la compagnie que de la réévaluation de ces offices inférieurs. Elles accompagnent en fait le divorce sociologique entre les deux institutions, la chambre des comptes devenant une étape ultérieure dans l'élévation sociale des familles de maire.

Tableau n ${ }^{\circ} 8$ - Ancienneté d'implantation nantaise des lignages de maires

\begin{tabular}{|c|l|c|c|c|}
\hline Générations & Lignages & Office & Élection & $\mathbf{1}^{\text {re }}$ mention \\
\hline \multirow{2}{*}{1} & Cornulier & $\mathrm{M}$ & 1569 & 1556 \\
& Fourché & $\mathrm{M}$ & 1597 & 1572 \\
& Pontual & $\mathrm{P}$ & 1656 & 1631 \\
\hline 2 & André & A.G. & 1591 & 1555 \\
& Huteau & $\mathrm{P}$ & 1658 & 1610 \\
\hline \multirow{2}{*}{3} & La Tullaye & $\mathrm{M}$ & 1629 & 1540 \\
& Juchault & $\mathrm{P}$ & 1642 & 1549 \\
& Monti & $\mathrm{M}$ & 1644 & 1547 \\
& Boux & $\mathrm{M}$ & 1648 & 1588 \\
\hline \multirow{2}{*}{4} & Le Lou & $\mathrm{M}$ & 1573 & 1503 \\
& Harouys & $\mathrm{P}$ & 1623 & 1530 \\
& Regnier & $\mathrm{A}$ & 1673 & 1558 \\
& Noblet & A.G. & 1690 & 1556 \\
\hline \multirow{2}{*}{5} & Le Lou & $\mathrm{M}$ & 1603 & 1503 \\
\hline 6 & Bernard & P & 1615 & 1449 \\
\hline
\end{tabular}

30. Arch. dép. de Loire-Atlantique, 25 J Nantes 16; Gaëtan d'AviAu DE TernaY, op. cit., p. 348 .

31. Idem, 25 J Nantes 14; ibidem, p. 311. 
Le calcul effectué, qui sous-estime peut-être la réalité par insuffisance de documentation sur certains lignages, révèle pourtant clairement l'essentiel, à savoir un profond enracinement urbain des lignages qui cumulent des charges dans les deux institutions. Une nette majorité (9/14) peut avancer une présence d'au moins trois générations, tandis qu'une belle minorité (5/14) peut se glorifier d'au moins quatre générations, c'est-à-dire d'une ancienneté quasi immémoriale. La répartition obéit à une logique complexe, au croisement de plusieurs influences parmi lesquelles le décollage dans les activités du grand commerce paraît plus déterminant que la date de saisie du poste de maire.

Tableau $\mathbf{n}^{\circ} 9$ - Ancienneté d'implantation nantaise des lignages d'échevins

\begin{tabular}{|l|l|c|c|c|}
\hline Générations & Lignages & Offices & Élections & 1re mention \\
\hline \multirow{2}{*}{1} & Monti & M & 1573 & 1547 \\
& Fourché & A, M & 1587 & 1572 \\
& Marcel & A & 1598 & 1587 \\
& Monnier & A & 1604 & 1587 \\
& Gallinière & A & 1637 & 1619 \\
\hline \multirow{2}{*}{2} & La Tullaye & A, M & 1589 & 1540 \\
& Juchault & A & 1601 & 1549 \\
& Davy & A & 1605 & 1549 \\
& Le Feuvre & A & 1611 & 1560 \\
& Grandamy & A & 1618 & 1582 \\
& Le Masle & A & 1625 & 1586 \\
& Barillier & A & 1645 & 1590 \\
& Forcheteau & G & 1661 & 1609 \\
\hline \multirow{2}{*}{3} & Adam & A & 1573 & 1499 \\
& Simon & A & 1574 & 1491 \\
& Cousin & A & 1583 & 1509 \\
& Maillard & A & 1584 & 1528 \\
& Madeleneau & A & 1623 & 1544 \\
& Le Breton & G & 1624 & 1513 \\
& Ragaud & A & 1636 & 1562 \\
& Juchault & A & 1645 & 1549 \\
\hline \multirow{2}{*}{4} & Macé & G & 1646 & 1537 \\
& Regnier & A & 1651 & 1558 \\
\hline \multirow{2}{*}{} & & & \\
\hline
\end{tabular}

Tout en se méfiant du biais statistique qui peut affecter le tableau par sous-information sur des lignages entrés à l'échevinage dans le dernier tiers du XvI ${ }^{\mathrm{e}}$ siècle, la répartition interne suggère que ce n'est sans doute pas un critère décisif dans la mesure où chaque catégorie est caractérisée par une assez bonne ventilation chronologique. Ici encore, ce sont les antécédents marchands qui assurent le mieux l'ancienneté nantaise. La dénivellation de puissance entre les lignages de maires et d'échevins est ici fort bien soulignée dans sa dimension temporelle. 
Guy SAUPIN

Tableau $\mathrm{n}^{\circ} 10$ - Environnement familial des officiers des comptes élus maires (1569-1675)

\begin{tabular}{|c|c|c|c|c|c|c|c|c|c|c|c|}
\hline Maire & Père & G.P.Pat & G.P.Mat & Onc.Pat & Onc.Mat. & F.Ai. & F.Cad. & B.F. & B.P. & B.F.Al. & B.FAIAl. \\
\hline Cornulier & Cap. & Cap. & & & & & & & & & \\
\hline Le Lou & Com. & Com. & & Com. & M.C. & & & & Com. & $\begin{array}{l}\text { G.Fi. } \\
\text { Sieur }\end{array}$ & $\begin{array}{c}2 \text { Com } \\
\text { R.Fo. }\end{array}$ \\
\hline André & Av. & & & & & & Av. & $\begin{array}{l}\text { A.R.P. } \\
\text { Sén. }\end{array}$ & A.C. & $\begin{array}{l}2 \text { M.C. } \\
\text { P.Mil. }\end{array}$ & \\
\hline Fourché & Sgr. & Sgr. & & & & & Av. & & & & \\
\hline Le Lou & M.C. & Com. & Com. & $\begin{array}{l}\text { G.Fi. } \\
\text { Sieur }\end{array}$ & $\begin{array}{l}\text { Com. } \\
\text { R.Fo. }\end{array}$ & & $\begin{array}{l}\text { C.Pa. } \\
\text { Sieur }\end{array}$ & $\begin{array}{c}3 \text { C.Pa } \\
\text { P.C. } \\
\text { G.EBF }\end{array}$ & $\mathrm{Fi}$ & & \\
\hline Bernard & Com. & Com. & Com. & $\begin{array}{l}\text { Com. } \\
\text { Av. }\end{array}$ & 2 Com & & Ch. C. & & $\begin{array}{l}\text { P.R.P. } \\
\text { S.P.P. }\end{array}$ & $\begin{array}{c}\text { Pr.P. } \\
\text { PG.E } \\
\text { B } \\
\text { Ch. C. }\end{array}$ & \\
\hline Harouys & S.PP. & G.Pa. & P.P. & $\begin{array}{l}\text { M.C. } \\
\text { Pr.Pa. } \\
\text { C.Pa }\end{array}$ & $\begin{array}{c}\text { P.P. } \\
\text { C.Pa. }\end{array}$ & Ch. C. & Pr.P. & P.C. & C.État & C.État & \\
\hline La Tullaye & M.C. & A.C. & & $\begin{array}{c}\text { M.C. } \\
\text { P. mil. } \\
\text { AG.C. } \\
\text { Prév. }\end{array}$ & & & & M.C. & Sieur & & \\
\hline Juchault & A.C. & Pr. C. & Com. & & & & $\begin{array}{c}\text { A.C. } \\
\text { PEBF }\end{array}$ & $\begin{array}{l}\text { M.C. } \\
\text { P.C. }\end{array}$ & Com. & & C.Pa. \\
\hline Monti & M.C. & M.C. & R. Fo. & $\begin{array}{c}\text { Av. } \\
\text { CG.B. }\end{array}$ & & & & RGF.B & Com. & $\begin{array}{l}\text { C. roi } \\
\text { Sieur }\end{array}$ & M.C. \\
\hline Boux & C.P. & D. M. & C.Pa. & $\begin{array}{c}\text { 2Pr.P. } \\
\text { R. Fo. } \\
\text { D. M. } \\
\text { Sgr. }\end{array}$ & S.Rég & & $\begin{array}{l}\text { A.C. } \\
\text { Sgr. }\end{array}$ & $\begin{array}{l}\text { C.P. } \\
\text { Sgr. }\end{array}$ & M.C. & $\begin{array}{l}\text { M.C. } \\
\text { Sieur }\end{array}$ & C.Pa. \\
\hline Pontual & Sgr. & & Sgr. & & & & & $\begin{array}{l}\text { T.E. B. } \\
\text { Gouv. }\end{array}$ & $\begin{array}{l}\text { C. Pa. } \\
\text { P.C. }\end{array}$ & $\begin{array}{l}2 \text { Cap. } \\
2 \text { Reli. }\end{array}$ & \\
\hline Huteau & M.C. & & & P.P. & & & & & C. Pa. & & \\
\hline Regnier & Com. & Com. & Com. & Av. & $5 \mathrm{com}$ & & & Com. & Célib. & & \\
\hline Noblet & R.TD. & Com. & & & & & & $\begin{array}{c}\text { L.cc.P } \\
\text { Sgr. }\end{array}$ & G.L.C. & $\begin{array}{l}\text { J.C.P. } \\
\text { Cap. }\end{array}$ & \\
\hline
\end{tabular}

Légende :

Parlement : P.Pa : président; C.Pa : conseiller; G.Pa. : greffier en chef

Comptes : P.C. : président M.C. : maître; A.C. : auditeur; AG.C. : avocat général; G.L.C. : garde des livres

Présidial : P.P. : président; S. sénéchal; L.cc.P. : lieutenant civil et criminel; J.C.P. : juge criminel; P.R.P. : procureur du roi; A.R.P. : avocat du roi

Autres gens justice : PEBF : procureur général des eaux, bois et forêts; $\mathrm{S}$. Reg. : sénéchal des régaires; Av. : avocat; Pr.P. : procureur au présidial

Finances : G.Fi : général des finances; R.Fo. : Receveur des fouages; RGF.B. : receveur général des finances de Bretagne; CG.B. : contrôleur général des finances de Bretagne; T.E.B. : trésorier des états de Bretagne; R.TD. : receveur du taillon et des décimes

Armée : Cap. : capitaine; P.Mil. : Prévôt militaire

Autres : Com : commerce; D.M. : docteur médecin; Ch.C : chanoine du chapitre cathédral; Sgr. : seigneur 
En saisissant globalement les orientations des lignages, on ne peut qu'être frappé par la diversification des cheminements suivis. En ne retenant que la filiation père (5) et grand-père (2), on souligne une fidélité aux Comptes pour cinq familles. En observant les choix professionnels des autres membres de la famille directe, on ne trouve que quatre familles intéressées par la cour souveraine, la plupart du temps à la génération de l'officier-maire, mais seuls les La Tullaye ${ }^{32}$ apparaissent dans ces deux groupes ${ }^{33}$. La même méthode appliquée aux membres alliés du lignage livre par contre neuf exemples, toujours principalement à propos de membres de la même génération.

Ainsi, nous constatons que les recherches d'alliances matrimoniales parmi les familles des Comptes proviennent autant de familles impliquées seulement par l'officier maire que de celles ayant déjà une présence plus lourde dans la compagnie. La quête d'appuis dans ce milieu d'anoblis en mue sociale explique largement cette inclination des familles nouvelles. N'en déduisons pas l'existence d'un modèle unique d'ascension robine. Pour souligner la fluidité des options dont disposent les lignages ambitieux, remarquons que seuls les La Tullaye se retrouvent dans les trois catégories précitées, avec présence d'officiers aux comptes dans l'ascendance directe, la parenté de sang immédiate et les familles alliées, les autres lignages les plus intéressés n'intervenant que dans deux d'entre elles ${ }^{34}$.

\section{Tableau $\mathbf{n}^{\circ} 11$-Situations sociales de la parenté proche des officiers élus maires}

\begin{tabular}{|l|l|l|l|l|l|}
\hline Mandat & Maire & Office & Père & Grand-père & Beau-père \\
\hline $1569-1570$ & P. Cornulier & Maître & Capitaine & Capitaine & \\
1573 & M. Le Lou & Maître & Com. Fin. & Com. & Com. Fin. \\
$1590-1591$ & P. André & Av. Général & Avocat & & Auditeur \\
$1597-1598$ & J. Fourché & Maître & Seigneur & Seigneur & \\
$1603-1605$ & Y. Le Lou & Maître & Com, Fi, Me & Com. Fin. & \\
$1615-1617$ & P. Bernard & Président & Commerce & Commerce & Proc. Roi Prés. \\
& & Président & Sén. Pr.Prés. & Greffier Par. & C. État \\
$1623-1625$ & C. de Harouys & Prés. \\
$1629-1631$ & R. La Tullaye & Maître & Maître & Auditeur & \\
$1642-1644$ & C. Juchault & Président & Auditeur & Proc. Cptes & Commerce \\
$1644-1647$ & Y. de Monti & Maître & Maître & Maître & Commerce \\
$1648-1650$ & M. Boux & Maître & Cons.Prés. & D. Méd. & Maître \\
$1657-1659$ & R. de Pontual & Président & Seigneur & Seigneur & Cons. Par. \\
$1659-1661$ & J. Huteau & Président & Maître & & veuve P. Cptes \\
$1673-1675$ & J. Regnier & Auditeur & Commerce & Commerce & Cons. Par. \\
$1690-1692$ & P. Noblet & Av. général & Rec. Taillon & Commerce & Garde Livres Cpt. \\
\hline
\end{tabular}

32. Héloïse MENARD, Ascension politique et sociale de la famile de La Tullaye, XVI ${ }^{-X V I T}$ siècles, mémoire de maîtrise, université de Nantes, 2000; Gaëtan d'AVIAU DE TERNAY, op. cit., p. 221-224.

33. Seuls les Juchault s'approchent de ce profil, en intégrant un office de simple procureur à la chambre, ce qui n'introduit pas dans la compagnie.

34. Il s'agit des Le Lou, Harouys, Juchault, Boux et André-La Tullaye, Bernard-Harouys, Monti-Bouriau, Boux-Ménardeau, Pontual-Rousseau, Noblet-Cassard. 
En se centrant sur l'ascendance directe, quelques lignages s'illustrent par un attachement plus particulier pour la chambre des comptes. Il en est ainsi des La Tullaye ${ }^{35}$ et des Monti ${ }^{36}$, à l'extrême limite des Juchault, présents sur trois générations. Pour le plus grand nombre, l'acquisition des offices de président ou de maître par ces notables devenus maires ne résulte pas d'un attachement particulier à la cour des comptes. La diversification des provenances est remarquable dès la génération paternelle, et naturellement encore plus à celle des grands-parents. Seuls trois officiers sur quinze cohabitent avec leur beau-père à la chambre. La comparaison des situations des pères et des beaux-pères invite toujours à relever la diversification. Le lien avec la chambre, toujours minoritaire, s'avère un peu moins net du côté des beaux-pères, et l'équilibre entre gens de justice et marchands mieux assuré du côté des pères, tandis que les beaux-pères sont plus facilement de simples propriétaires de sieuries ou de seigneuries.

\section{Tableau $\mathbf{n}^{\circ} 12$ - L'environnement familial des officiers des comptes élus échevins}

\begin{tabular}{|c|c|c|c|c|c|c|c|c|c|c|c|}
\hline Échevin & Père & G.P.pat. & G.P.mat. & Onc.pat. & Oncmat. & F.Ai. & F.Cad. & B.F. & B.P. & B.F.Al. & B.FAIAl. \\
\hline Monti & C. Flo. & & & & & & & & G. Pr. & & \\
\hline Adam & Com. & & Apot. & & & & & & & & \\
\hline \multicolumn{12}{|l|}{ Simon } \\
\hline Cousin & $\begin{array}{c}\text { No.PC } \\
\text { C.Pa }\end{array}$ & & & & & & & & $\begin{array}{l}\text { No.PP } \\
\text { MPEF }\end{array}$ & & \\
\hline Maillard & Apot. & & & & & & & & Com. & 2 Com. & \\
\hline Fourché & Sgr. & Sgr. & & & & & Av. & & Com. & & \\
\hline La Tullaye & A.C. & Fi. & & & & $\begin{array}{l}\text { M.C } \\
\text { P. mil }\end{array}$ & & & Com. & Sieur & \\
\hline Marcel & & & & & & & & & R. Do. & $\begin{array}{l}\text { G. Fi. } \\
\text { R. Fo. }\end{array}$ & PREF \\
\hline Juchault & Pr. C. & & & & & & & & Com. & & \\
\hline Monnier & Com. & & & & & & & & Com. & Com. & \\
\hline Davy & Com. & & & & & & C.Do. & & Com. & & \\
\hline Le Feuvre & Av. & & & & & & J.cri. & & & & A.C. \\
\hline Grandamy & Com. & & & & & & & & & & \\
\hline Madeleneau & Com. & Com. & Com. & Com. & & Com. & $\begin{array}{l}\text { A.C } \\
\text { R.Fo. }\end{array}$ & & Com. & $\begin{array}{l}\text { A.C. } \\
\text { C.Tr. }\end{array}$ & Sieur \\
\hline Le Breton & Com. & & & & & C.P. & & & A.C. & & A.C. \\
\hline Le Masle & A.C. & & & & & & & & Gr. C. & $\begin{array}{l}\text { Av. } \\
\text { C.P. }\end{array}$ & \\
\hline Ragaud & CG.Pr & Com. & PF.R & & $\begin{array}{l}\text { P.P. } \\
\text { Av. } \\
\text { C.P. }\end{array}$ & Sieur & $\begin{array}{l}\text { Rect. } \\
\text { CG.Pr }\end{array}$ & & & & A.C. \\
\hline
\end{tabular}

35. Arch. dép. de Loire-Atlantique, 2 E 1501 Cette généalogie comprend quelques erreurs. René Kerviler, op. cit., t.11, p. 696-699. Voir d'abord Gaëtan d'AviAU dE TERNAY, op. cit., p. 221-224.

36. Henri Frotier de LA Messeliere, op. cit., t. 4, p. 97-106; Jules Mathorez, « Les Italiens à Nantes et dans le Pays nantais ", Bulletin italien, Bordeaux, 1913, t. 13, p. 112-129. 
Les officiers de la Chambre et le corps de ville

\begin{tabular}{|c|c|c|c|c|c|c|c|c|c|c|c|}
\hline Échevin & Père & G.P.pat. & G.P.mat. & Onc.pat. & Onc.mat. & F.Ai. & F.Cad. & B.F. & B.P. & B.F.Al. & B.FAlAl. \\
\hline Gallinière & & & & & & $\begin{array}{c}\text { Sieur } \\
\text { Av. }\end{array}$ & & & P.P. & Sieur & $\begin{array}{l}\text { Sieur } \\
\text { Sieur }\end{array}$ \\
\hline Juchault & A. C. & Pr. C. & Com. & & & P.C. & PREF & $\begin{array}{l}\text { M.C. } \\
\text { P.C. }\end{array}$ & & A.C. & \\
\hline Barillier & Fi.Mo. & Av. & Com. & & $\begin{array}{l}\text { R. Fo } \\
\text { A.C. } \\
\text { A.R.P } \\
\text { C.P. } \\
\text { Com. }\end{array}$ & & A.C. & AR.P. & Av. & & \\
\hline Macé & Ga. C. & Gr.P. & Com. & $\begin{array}{c}\text { A.C. } \\
\text { P.P. } \\
\text { Gr.Pv. } \\
\text { Alloué } \\
\text { Pr. Pa }\end{array}$ & & & & & & Com. & $\begin{array}{l}\text { Av. } \\
\text { Sgr. }\end{array}$ \\
\hline Regnier & Com. & Com. & & & Av. & 5 Com. & & Com. & Céli. & & \\
\hline Forcheteau & Gr.P. & & & & & $\begin{array}{c}\text { Gr.P } \\
\text { CFi.B }\end{array}$ & & MPEF & $\begin{array}{c}\text { Com. } \\
\text { Mis. }\end{array}$ & & Com. \\
\hline
\end{tabular}

Légende :

Comptes : P.C. : président; M.C. : maître; A.C. : auditeur; Gr.C. : greffier; Ga.C. : garde des livres

Présidial : J. cri : juge criminel; C.P. : conseiller; AR. P. : avocat du roi ; Gr.P. : greffier Gens de justice : Av. : avocat; Pr. : procureur; MPEF : maître particulier des eaux et forêts ; PREF : procureur du roi aux eaux et forêts ; PF. R : procureur fiscal aux réguaires ; Gr.Pv. : greffier à la prévôté

Finances : G. fi : général des finances; R. Do : receveur du domaine royal; R. Fo : receveur des fouages; CG.P. : contrôleur général à la prévôté

Divers : C. Flo. : conseiller ducal à Florence; G. Pr. : grand prévôt Anjou; P. mil : prévôt militaire

Le décalage de puissance souvent observé entre familles de maire et d'échevins se vérifie une fois encore dans cette analyse de la strate sociale pourtant supérieure occupant ces charges secondaires du bureau de ville. Alors que le nombre de cas analysés est supérieur (23 officiers échevins contre 15 officiers maires), les références à la chambre des comptes s'avèrent nettement moins nombreuses. Seules quatre familles sont citées pour l'ascendance directe (La Tullaye, Le Masle ${ }^{37}$, Juchault et Macé ${ }^{38}$ ), trois pour la parenté directe des oncles et frères (La Tullaye, Juchault, Barillier ${ }^{39}$ ) et six pour des membres attachés par alliance. Parmi ces dernières, quatre renvoient à des familles qui n'ont placé que l'officier échevin au bureau de ville, ce qui souligne combien ici la recherche d'appuis supérieurs est encore plus déterminant que pour les familles de maire dont la puissance sociale est mieux établie. Au total, seuls les La Tullaye et les Juchault ${ }^{40}$ sont fortement infiltrés dans la compagnie des comptes. Remarquons que ce sont aussi des lignages de maire déjà étudiés précédemment.

37. Arch. dép. de Loire-Atlantique, 25 J Nantes 11; Gaëtan d'AvIAU DE TERNAY, op. cit., p. 250. 38. Idem, 25 J Nantes 12 ; ibidem, p. 272-275.

39. Id., $25 \mathrm{~J}$ Nantes 2; ibid., p. 15.

40. Id., 25 J Nantes 9; ibid., p. 206-208. 
Guy SAUPIN

Tableau $n^{\circ} 13$-Situation sociale de la parenté proche des officiers élus échevins

\begin{tabular}{|c|c|c|c|c|c|}
\hline Mandat & Échevin & Office & Père & Grand-père & Beau-père \\
\hline $1573-1575$ & B. de Monti & M. & Cons. duc Flor & & Prévôt Anjou \\
\hline $1573-1575$ & A. Adam & A. & Marchand & & \\
\hline $1574-1576$ & F. Simon & A. & & & \\
\hline 1583-1585 & J. Cousin & A. & N. PP. C. Parle. & & N. PP. MEPF. \\
\hline $1584-1586$ & E. Maillard & A. & Apothicaire & & Marchand \\
\hline 1587-1589 & J. Fourché & A., M. & Seigneur & Seigneur & Marchand \\
\hline 1589-1591 & J. La Tullaye & A., M. & Auditeur & Finances & Marchand \\
\hline 1598-1601 & G. Marcel & A. & & & Finances \\
\hline $1601-1604$ & M. Juchault & A. & Proc. comptes & & Marchand \\
\hline $1604-1607$ & P.Monnier & A. & Marchand? & & Marchand \\
\hline $1605-1608$ & P.Davy & A. & Marchand? & & Marchand \\
\hline $1611-1614$ & A. Le Feuvre & A. & Avocat & & \\
\hline $1618-1621$ & F. Grandamy & A. & Md. Me monnaie & & \\
\hline $1623-1626$ & P. Madeleneau & A. & Marchand draps & Marchand & Marchand \\
\hline $1624-1627$ & J. Le Breton & G. & Marchand & & Auditeur \\
\hline $1625-1628$ & P. Le Masle & A. & Auditeur & & \\
\hline $1636-1637$ & J. Ragaud & A. & C.G. Prévôté & Marchand & \\
\hline $1637-1640$ & M. Gallinière & A. & & & Proc. Présidial \\
\hline $1645-1648$ & C. Juchault & A. & Auditeur & Proc. Cptes & Sieur \\
\hline $1645-1648$ & D. Barillier & A. & Fin., M. monnaie & Avocat & Avocat \\
\hline $1646-1649$ & G. Macé & G. & G. livres Comptes & Greffier Prés. & Marchand drap \\
\hline $1651-1654$ & J. Regnier & A. & Marchand & Marchand & Célibataire \\
\hline $1661-1664$ & M. Forcheteau & G. & Greffier Prés. & & Md., Miseur \\
\hline
\end{tabular}

En ne retenant que les situations socioprofessionnelles les plus proches, on découvre combien ces familles d'officiers des Comptes échevins sortent naturellement du commerce ou de la judicature, les deux grands milieux classiquement pourvoyeurs de l'élite urbaine. Dans cette dimension parentale étroite, l'accès à la cour souveraine ne concerne que fort peu de familles. Soulignons un léger déséquilibre entre la filiation de parenté directe, plus équilibrée, et la sélection des alliances, un peu plus appuyée sur le monde marchand. Cette présence plus significative d'un environnement familial plus relié au commerce crée une différence avec le profil observé à propos des lignages de maire où cet horizon paraît rejeté plus loin en arrière.

La comparaison de l'ancienneté d'implantation nantaise des lignées fournissant à la chambre des comptes et au bureau de ville tourne nettement à l'avantage des familles de maires, même si certaines familles échevinales peuvent se prévaloir d'une ancienneté respectable. Pourtant, lorsque plus de la moitié des familles de maires (10/15) font valoir trois générations et plus, plus de la moitié des familles échevinales (13/23) n'ont pas cet avantage. Si les édiles qui représentent la première génération de présence sont venus à Nantes pour entrer à la chambre des comptes, ceux 
de la seconde génération ne sont reliés à cette compagnie que pour une minorité d'entre-eux, malgré la règle de l'anoblissement graduel. Cette évaporation marque bien comment la recherche de l'échevinage s'envisage plutôt antérieurement à un maintien prolongé dans la cour souveraine, par suite du différentiel de prestige entre les deux institutions. Cette remarque vaut beaucoup plus pour les familles échevinales que pour les familles de maires, à cause du degré de puissance sociale qui en sépare le plus grand nombre.

Même si l'échantillon est limité et particulier, toutes ces remarques invitent à souligner la relative souplesse qui caractérise le renouvellement de l'élite sociale nantaise. Si l'ancienneté est reconnue, l'intégration rapide des nouveaux arrivés n'est pas impossible. L'enchaînement des activités professionnelles au sein des trajectoires lignagères est loin de se réduire à un modèle unique. Tout indique que dans une grande ville où les fonctions administrative et commerciale s'inscrivent dans une concurrence équilibrée se maintient une élite ouverte capable d'accueillir en son sein tous les candidats pouvant faire valoir aisance et réputation.

Contrairement aux parlementaires des grandes villes françaises ${ }^{41}$, les officiers de la chambre des comptes de Bretagne n'ont pas refusé de siéger au sein du corps de ville de Nantes. En cela, ils se comportent comme les officiers d'autres chambres du royaume, comme à Aix-en-Provence ${ }^{42}$ ou Montpellier ${ }^{43}$. À Paris, si le prestige de la charge de prévôt des marchands demeure intact, les responsabilités échevinales ne sont pas dédaignées jusqu'à la Fronde, même si les officiers des cours souveraines ne représen-

41. Dans les capitales parlementaires, la cour souveraine surveille de près la gestion d'un corps de ville souvent abandonné à la bourgeoisie juridique seconde et aux marchands, avec un règlement destiné à garantir un bon équilibre sociologique. La jurade de Bordeaux comprend deux nobles, deux avocats et deux marchands; le capitoulat de Toulouse, deux nobles, deux avocats, deux procureurs et deux marchands; l'échevinage de Dijon recrute dans les mêmes catégories, tandis que le poste de maire de Besançon est monopolisé par les avocats au XVIII ${ }^{\mathrm{e}}$ siècle; Laurent CosTE, "La jurade de Bordeaux au $\mathrm{XVII}^{\mathrm{e}}$ siècle ", dans Josette PONTET (dir.), Des hommes de pouvoir dans la ville, XIV -XX $^{e}$ siècles, France, Allemagne, Angleterre, Italie, Bordeaux, CESURB, 1999, p. 257-287; Robert A. SCHNEIDER, Public Life in Toulouse, 1463-1789. From municipal Republic to Cosmopolitan City, Londres, 1990; Idem, "Les parlementaires dans la vie municipale et sociale " dans Jacques Poumarede et Jack Thomas (éd.), Les parlements de province. pouvoirs, justice et société, Toulouse, 1996, p. 662 et suiv.; Gaston RouPNEL, La ville et la campagne au XVII siècle. Étude sur les populations du pays dijonnais, Paris, 1955, p. 164-166; Maurice GRESSET, Gens de justice à Besançon (1674-1789), Paris, 1978.

42. Jacqueline Dumoulin, Le consulat d'Aix-en-Provence. Enjeux politiques, 1598-1692, Dijon, EUD, 1992.

43. Frederick M. IRVINE, "From Renaissance to Ancien Régime Capital : Montpellier, C.1500-C. 1600 ", dans Philip BenEdict (ed.), Cities ans Social Change in Early modern France, Londres, 1989, p. 105-133. L'auteur montre comment l'édit de 1547 n'a pas été respecté et comment le désordre lié aux guerres de religion a favorisé l'ascension des gens de justice au sein du consulat. Les officiers des comptes se sont naturellement intéressés au poste de premier consul et secondairement au second chaperon dans un exécutif à six charges. 
tent qu'environ $7 \%$ des promus qui viennent surtout du monde judiciaire inférieur, des finances et surtout du commerce supérieur ${ }^{44}$.

Cette attitude a progressivement disparu sous le règne de Louis XIV, rapidement pour les charges d'échevins, avec plus d'hésitation pour le poste de maire. La progression de la centralisation administrative et l'amputation partielle des privilèges d'anoblissement municipal ont déprécié ce type de service civique aux yeux d'une strate sociale qui se considérait au sommet de la pyramide sociale nantaise ${ }^{45}$. Pourtant, la chronologie suggère que la procédure de divorce, engagée à l'initiative des officiers, a précédé cet appesantissement du contrôle royal qui ne se fait sentir qu'à la fin des années 1660. Peut-être faut-il souligner l'importance d'un malaise au sein d'une compagnie officière qui ressent négativement les effets de la nouvelle gestion par intendant sur les officiers de finances ${ }^{46}$ et la politique de promotion de l'honorabilité marchande par logique mercantiliste ${ }^{47}$ ? La politique contradictoire de la monarchie au cours du second tiers du $\mathrm{XVII}^{\mathrm{e}}$ siècle, en ne soutenant pas les officiers des Comptes au niveau où les plaçait le corps politique nantais tout en impulsant une réforme pour mieux les positionner comme candidats, n'a pu que semer le doute et favoriser cette désertion de la vie publique ${ }^{48}$.

Ce réflexe d'aristocratisme urbain, nourri de l'imitation du comportement parlementaire, n'est pas sensible partout à la même époque dans des institutions royales de niveau comparable. Les officiers du bureau de finances de Tours, figurant assurément la partie la plus marquante de l'élite urbaine, manifestent toujours de l'intérêt pour l'échevinage, même si une certaine érosion affecte le $\mathrm{XVIII}^{\mathrm{e}}$ siècle $^{49}$.

44. Robert Descimon, "L'échevinage parisien sous Henri IV (1594-1609). Autonomie urbaine, conflits politiques et exclusives sociales ", dans Neithard Bulst et Jean-Philippe GENET (éd.), La ville, la bourgeoisie et la genèse de l'État moderne, Paris, CNRS, 1988, p. 136. L'auteur précise l'évolution en trois phases (1560-1587, 1594-1609, 1611-1647) pour plusieurs catégories. Cours souveraines : 3,5, 6,3, 6,7 \%. Autres cours : 15,8, 25, 21,3\%. Finances : 19,3, 15,6, 10,7 \%. Basoche : 22,8, 21,9, 14,7 \%. Marchandise : 36,8, 28,1, 44 \%.

45. Guy SAuPIN, Nantes au XVII siècle..., op. cit., p. 262-266.

46. Même si la Bretagne demeure un pays d'états, elle voit arriver un commissaire départi en 1647, lequel risque de déstabiliser l'activité des trésoriers de France qui sont intégrés à la compagnie des Comptes.

47. Les années 1640 sont particulièrement révélatrices de ce nouveau climat soutenu à Nantes par le maréchal de La Meilleraye. En 1641, le bureau de ville décide la construction de la première bourse de commerce; en 1645, le maréchal lance une compagnie à monopole vers l'océan Indien; en 1646, le carme Jean Éon, d'une famille marchande malouine, publie son célèbre livre, Le commerce honorable, de pure inspiration mercantiliste.

48. L'abandon de la réforme, qui structurellement imposait la présence d'officiers des comptes dans les listes présentées au pouvoir royal pour les nominations au bureau de ville, au bout de dix ans d'exercice, en 1654, n'a pu qu'approfondir un malaise préexistant, accélérant ainsi une logique de séparation.

49. Claude PETITFRERE, "Les officiers dans le corps de ville de Tours aux XVII ${ }^{\mathrm{e}}$ et XVIII ${ }^{\mathrm{e}}$ siècles ", dans Michel CASSAN, Les officiers moyens..., op. cit., p. 121-138. Sur les 92 maires en fonction de 1589 à 1789, 70 sont officiers royaux dont 31 du présidial et 23 du bureau des 
Dans cette première modernité où l'appartenance à la cour souveraine n'apparaît pas contradictoire avec l'animation du corps de ville, les responsabilités obtenues illustrent une fois de plus le clivage social interne entre les offices supérieurs de maîtres et de présidents ouvrant au poste de maire et ceux d'auditeurs orientant massivement vers l'échevinage. Cette dénivellation se traduit par ailleurs par un moindre investissement des Comptes par les familles échevinales pourtant attirées par la cour. La diversité des itinéraires suivis par toutes ces lignées en ascension sociale plaide pour l'image d'une élite urbaine ouverte, juste pendant d'un attachement à la hiérarchie des ordres volontiers définie comme éternelle ${ }^{50}$. La variété de l'enracinement nantais des familles considérées renforce cette impression de fluidité.

La concomitance des exercices de fonction publique étatique et municipale renvoie à un modèle politique où les notables n'ont pas encore ressenti le basculement vers une monarchie de type administratif. Tant que les derniers feux du modèle de la monarchie mixte des " bonnes villes " ne se sont pas encore éteints, le service d'une puissante communauté d'habitants porte encore suffisamment de prestige pour sembler valorisant à des officiers de cour souveraine de seconde catégorie. Lorsque la procédure judiciaire arbitrale cède la place à la contrainte par commandement du gouvernement, les mêmes officiers rejoignent les parlementaires dans leur refus de compromission sociale dans un corps de bourgeoisie affaibli. La noblesse robine, en s'excluant volontairement des corps de ville, repousse ainsi la principale structure institutionnelle qui lui aurait permis de préserver ce rôle de représentation de la population qu'elle continuait par ailleurs à inscrire dans l'essence même du second ordre. Par là, elle s'exposait au risque de l'évanescence de ce privilège politique. L'investissement nobiliaire massif des corps de ville espagnols aux XVI ${ }^{\mathrm{e}}$ et $\mathrm{XVII}^{\mathrm{e}}$ siècles ${ }^{51}$, dans le cadre de la préservation d'une gestion étatique décentralisée, s'inscrit ainsi comme une alternative à l'évolution française ${ }^{52}$.

finances. Pour ces derniers, il existe un contraste réel entre les XVII ${ }^{\mathrm{e}}$ et XVIII ${ }^{\mathrm{e}}$ siècles : 9 pour

1589-1650, 11 pour 1651-1700, 3 pour 1701-1789. Il en est de même pour les charges d'échevin. 1600-1650 : sur 53 promus, 30 officiers dont 11 du présidial, 3 du bureau des finances et 4 de l'élection. 1651-1700 : sur 54 élus, 30 officiers dont 11 du présidial, 8 du bureau des finances et 3 de l'élection. 1701-1789 : sur 103 élus, 40 officiers dont 15 du présidial et $4 \mathrm{du}$ bureau des finances.

50. Afin de replacer ces familles municipales attachées à la chambre des comptes dans un ensemble plus vaste, voir Guy SAUPIN, "Considération sociale : les choix socioprofessionnels des familles des maires de Nantes du XVII ${ }^{\mathrm{e}}$ siècle sur le temps long ", dans Josette Pontet (éd.), À la recherche de la considération sociale, Bordeaux, MSHA, 1999, p. 105-119; Idem, "Les stratégies lignagères des familles échevinales nantaises du XVII siècle " dans Pouvoirs des familles, familles de pouvoir, Colloque de Toulouse, octobre 2000, Actes à paraître.

51. José Manuel de Bernardo Ares, Jésus Manuel Gonzalez Beltran, (ed.), La administracion municipal en la Edad moderna, Cadiz, Universidad de Cadiz y Asociacion espanola de Historia moderna, 1999. 


\section{RESUME}

Jusqu'à la transformation des fonctions municipales en offices vénaux, certains maîtres et présidents des Comptes s'intéressent au poste de maire de Nantes tandis que des auditeurs ne dédaignent pas de siéger comme échevins. Le divorce, total au $\mathrm{XVIII}^{\mathrm{e}}$ siècle, s'amorce plus vite dans l'échevinage qu'au niveau de la présidence du bureau de ville. Dans le dernier tiers du $\mathrm{XVI}^{\mathrm{e}}$ siècle, la conjoncture politique pèse lourd, tant dans la recherche du loyalisme monarchique que dans la rébellion ligueuse. Au XvII ${ }^{\mathrm{e}}$ siècle, si le pouvoir royal suit de près l'expression du corps politique nantais pour le choix des maires, il soutient mal les auditeurs pour les postes d'échevins, précipitant sans doute une rupture par ailleurs portée par une réaction de type aristocratique. Dans les stratégies familiales d'ascension sociale, se retrouve le décalage structurel entre les deux niveaux du bureau de ville, tant dans l'ancienneté d'enracinement urbain que dans l'ampleur de puissance sociale, même si les parcours demeurent fort diversifiés.

\section{ABSTRACT}

Until the transformation of the municipal functions into venal offices, some masters and presidents of the Chambre des comptes are interested in the office of mayor of Nantes and some listeners hope for being municipal magistrates. The breakup, which is complete during the XVIII ${ }^{\text {th }}$ century, starts more quickly in the échevinage than for the presidency of the city. In the last third of the XVIt century, the political economic situation is essential, as well in the search of monarchical loyalty as in the rebellion of the Ligue. During the XVII century, if the monarch follows closely the choices of party in power of Nantes for the appointement of the mayors, it badly supports the listeners for the posts of municipal magistrates, undoubtedly precipitating a rupture carried by a reaction of an aristocratic type. Now, in the family strategies of social rise, there is a gap between two models of magistrates, as well in seniority in rank as in social power.

52. Guy SAUPIN, "Stratification sociale et désignation des corps de ville dans les grandes villes françaises (XVI ${ }^{\mathrm{e}}$-XVIII ${ }^{\mathrm{e}}$ siècles) ", dans Martine LAMBERT-GEORGES, (éd.), Les élites locales dans l'Espagne moderne du XVTe au XIX siècle, Paris, CNRS, 1993. 